\title{
Decision Accuracy and the Role of Spatial Interaction in Opinion Dynamics
}

\author{
Colin J. Torney · Simon A. Levin • Iain D. Couzin
}

\begin{abstract}
The opinions and actions of individuals within interacting groups are frequently determined by both social and personal information. When sociality (or the pressure to conform) is strong and individual preferences are weak, groups will remain cohesive until a consensus decision is reached. When group decisions are subject to a bias, representing for example private information known by some members of the population or imperfect information known by all, then the accuracy achieved for a fixed level of bias will increase with population size. In this work we determine how the scaling between accuracy and group size can be related to the microscopic properties of the decision-making process. By simulating a spatial model of opinion dynamics we show that the relationship between the instantaneous fraction of leaders in the population $(L)$, system size $(N)$, and accuracy depends on the frequency of individual opinion switches and the level of population viscosity. When social mixing is slow, and individual opinion changes are frequent, accuracy is determined by the absolute number of informed individuals. As mixing rates increase, or the rate of opinion updates decrease, a transition occurs to a regime where accuracy is determined by the value of $L \sqrt{N}$. We investigate the transition between different scaling regimes analytically by examining a well-mixed limit.
\end{abstract}

Keywords Opinion dynamics · Collective behavior

\section{Introduction}

Collective decisions in many natural, social, and economic settings often take place in the absence of any explicit centralized control. Social pressures and individual knowledge or preferences cause opinions to fluctuate and diffuse, until a single dominant opinion emerges and a consensus is reached. Sociality (or conformity) is an integral part of this decisionmaking process as it potentially improves both decision accuracy and speed. It further represents an individually adaptive behavior, since useful information may be gleaned from the 
actions of others [1], while considerable costs may be associated with adopting a minority view [2].

Unfortunately, even simple social interactions lead to highly complex collective behaviour [3]; therefore, in order to understand the dynamics of large numbers of interacting entities, investigators have employed techniques borrowed from statistical physics [4]. The systems studied using this approach have ranged from the movement decisions of simple organisms [5, 6] to the complex behavior of human societies [7]. While this approach has attracted criticism for its over-simplification of individual agents, its strength lies in creating tangible connections between different levels of social or biological organization. In this way emergent, macroscale properties can be related to the underlying microscale behaviors [8]. Through the study and classification of these simplified models, the role of generic features (for example, dimensionality [9] or topology [10]) may be understood and ultimately related to empirical data and the behavior of real-world systems.

A significant macroscale property in many decision-making systems is the level of accuracy achieved when one outcome is preferred or optimal, i.e. the probability the correct outcome will be realized for a single instance of the decision process. This collective accuracy is influenced by individual behavior and the nature of social interaction, system size, and the degree of available information. The most notable relation between these quantities is the tendency for accuracy to increase with group size; a property that has been demonstrated by theoretical and empirical work related to both humans and animals [11-18]. This 'more is better' relationship is well understood in the case of structured decisions, when information is aggregated through formal voting procedures [11]; however when social imitation and local interaction drive collective decisions, the nature of the scaling of decision accuracy with group size remains unclear.

To address this deficit, we study a binary-choice model (intended as a simplified version of animal movement and direction choice) in order to better understand the relation between accuracy and group size. We assume one option represents the correct choice and investigate the level of accuracy achieved when only a fraction of individuals are provided with this information. Leadership roles are dynamic; at each time step a fraction $(L)$ of individuals are designated as leaders and their behavior is altered so that they will not switch away from the correct opinion. We may conceptualize our approach either as a first approximation to fixed leadership roles (as employed in [16, 19-21]) where we have neglected the correlation between leadership status and current opinion, or alternatively, as a model of imperfect information where individuals make choices based on a personal estimate, such as in Condorcet's jury theorem [11] (two discrete choices) or the 'many wrongs' hypothesis [14] (a continuous directional choice).

The structure of the article is as follows. Firstly, we embed the process in a twodimensional spatially-explicit domain to more closely match the approach of [16] and the mobile animal groups that are our primary focus. We show numerically that scaling depends on the individual-level behavioral rules and degree of social mixing. In the analysis that follows we retain only the key properties that determine the scaling relation between system size and accuracy. By switching to a more abstract domain we are able to identify a non-system specific connection between accuracy scaling and individual behavior, which in principle may apply to various forms of interaction topology.

\section{A Spatially-Explicit Model of Opinion Dynamics}

Many models of opinion formation consider agents deciding between two options. This choice may relate to discrete directions, a choice between two different priorities such as 
food or shelter, or more complex options such as opposing political viewpoints, or whether to buy one type of technology over another. Historically binary-choice models have been employed due to their tractability, and their origins in classic models of statistical physics such as the Ising model. Recently, these models have proved to be effective in understanding the behavior of real animal groups, as demonstrated in [19]. We therefore employ this approach and investigate the behavior of a binary choice model, embedded in two-dimensional space with nearest neighbor social interaction.

Animal groups are dynamic and mobile [22] with frequently changing social contacts, a property that may strongly influence the underlying decision-making dynamics. To introduce a spatial dynamic to our model, individuals are embedded into a chaotic mixing flow defined on a unit torus. While this is a stylized version of genuine dynamics, it captures the chaotic nature of the interactions between large numbers of mobile entities.

Following [23] we use an alternating sinusoidal shear flow for our advecting force, defined as

$$
\begin{aligned}
& \frac{1}{v} \frac{d x}{d t}=\Theta\left(\frac{T}{2}-t \bmod T\right) \cos (2 \pi y) \\
& \frac{1}{v} \frac{d y}{d t}=\Theta\left(t \bmod T-\frac{T}{2}\right) \cos (2 \pi x)
\end{aligned}
$$

where $x, y$ are the spatial coordinates of an individual, $\Theta(z)$ is the Heaviside step function, $v$ determines the speed of advection and $T$ defines the frequency with which the flow oscillates. For simplicity, we set $T$ to $(2 v)^{-1}$, so that there exists a single connected region and the flow is ergodic with no isolated islands [23]. While results are not dependent on the exact nature of the mixing flow employed, this ergodicity condition is essential for the population to remain cohesive; otherwise islands of interacting individuals will form and the effective system size will be reduced.

As the units for space and time are arbitrary we set the domain to be length 1 meter and the time scale on which a social observation occurs to be 1 second. For illustration, snapshots of the system when individuals are all initially located on the left of the domain are shown in Fig. 1 (individuals are shown as points regardless of their opinion).

Within this environment individuals make observations of their instantaneous nearestneighbor. The opinion state of the neighbor is noted and a tally is kept of the number of observations of each opinion. In this way a sample of the population opinion is built up over time. At each time step each individual decides to update its current opinion with a certain probability, denoted by $\gamma$. Reducing this probability results in longer times between opinion updates and therefore a larger sample of the population.

This decision rate therefore defines a memory length for each individual; the time over which an individual is able to keep a record of its interactions. Unlike models that occur on the lattice or across a network, the instantaneous configuration does not define the interaction topology, instead this topology is built up over time and is a function of the recent history of the spatial dynamics.

If an individual decides to update its current state, it will switch to the majority opinion observed within its record of samples. However, an individual will not switch to the incorrect option if it is designated as a leader. Leadership roles are dynamically allocated with each individual having a probability $L$ of being informed at each time step. We are principally investigating the effect the parameter $L$ has on the overall accuracy. As there exist two absorbing states, representing unanimous consensus for either opinion, accuracy is defined as the frequency with which global consensus for the correct state is reached. 

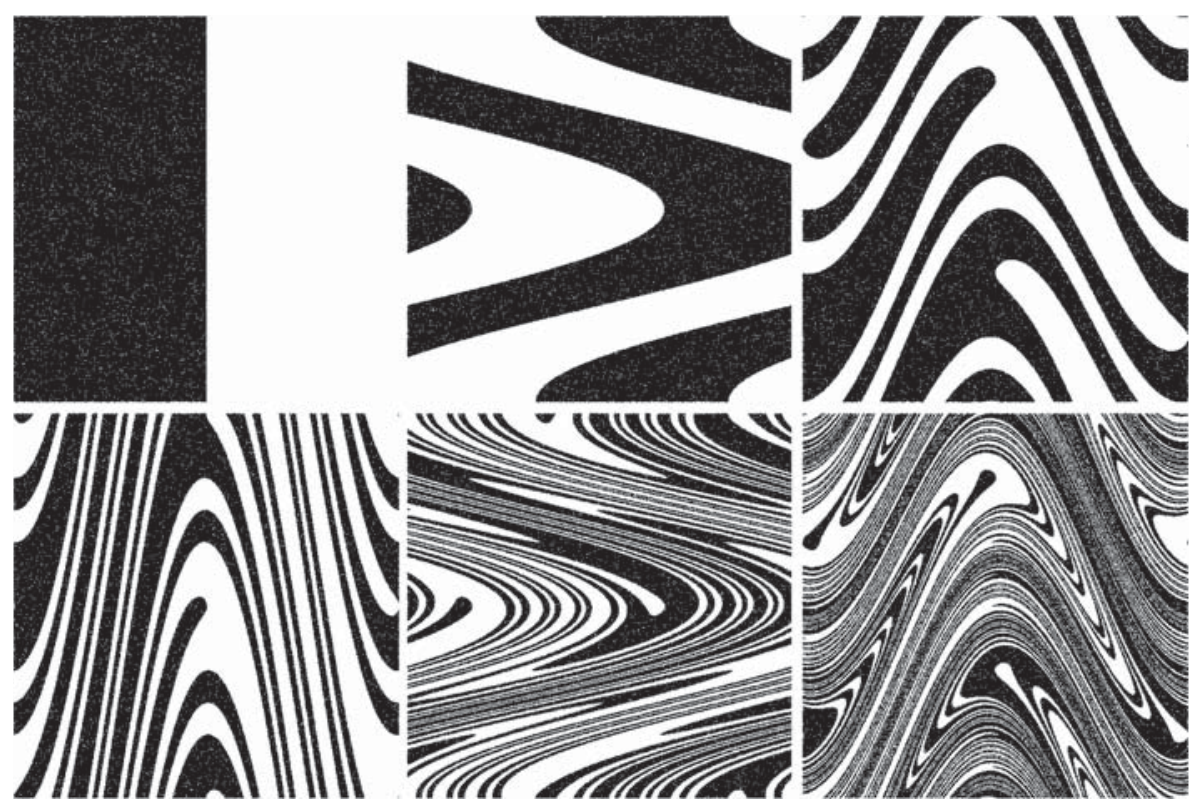

Fig. 1 Illustration of the chaotic mixing defined by Eq. (1). $N=524,288$. Individuals initially located on the left of the domain are advected. No opinion changes take place within this simulation. The speed $v$ is set to $0.67 \mathrm{~m} / \mathrm{s}$ and snapshots are shown every second. Time progresses left to right, upper panel to lower

To summarize the dynamics, we outline here the algorithm used.

- Individuals are initially distributed at random within the unit torus, with half of all individuals holding the correct opinion $(+1)$ and half adopting the incorrect view (denoted -1 ).

- The position of each individual is updated according to the advection velocity defined in Eq. (1).

- Each individual observes its nearest neighbor and increments or decrements a counter accordingly. This effectively builds a sample of the population opinion.

- With probability $\gamma$ an individual decides it will update its state.

- If according to its personal counter more individuals are in the +1 state, the individual will adopt this state (in the event of a tie, no change in opinion occurs).

- If according to its estimate, more individuals are in the -1 state, the individual will switch to this state with probability $(1-L)$. Otherwise it is considered an informed individual and will not switch.

- If all individuals hold the same opinion the trial ends. If consensus has not been reached positions are updated and the process repeats.

\subsection{Numerical Results}

Our simulations demonstrate that as the population size increases relatively fewer informed individuals are required to maintain the same level of accuracy. This effect is illustrated in Figs. 2A and 2B, which show accuracy versus informed population size for different values of spatial mixing and individual update rate. (Accuracy has been rescaled from 0 (random choice) to 1 (always correct) as in [16].) In each inset of these figures, the accuracy attained as a function of the fraction of informed individuals for different population sizes is 
(A)

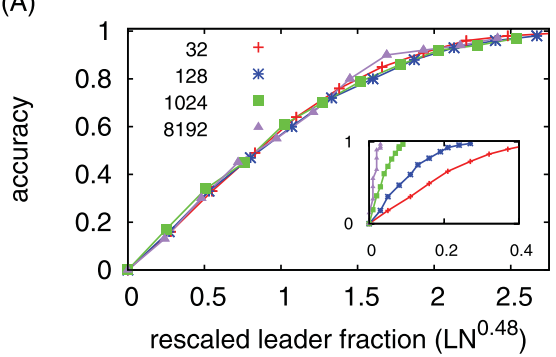

(B)

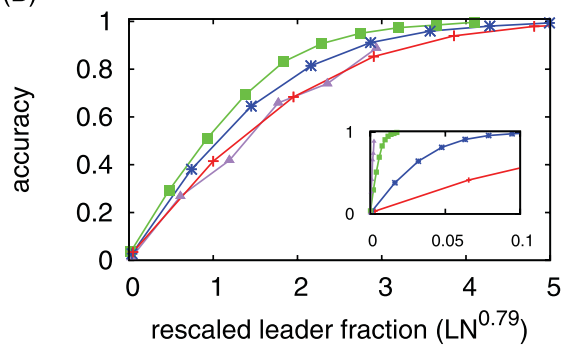

Fig. 2 Spatial simulations (A) Accuracy as a function of population size for fast mixing and slow updates, $v=2, \gamma=0.1$. Main figure shows accuracy as a function of the rescaled fraction of informed individuals $L N^{-\alpha}$ where $\alpha=-0.48$. Inset: Accuracy as a function of $L$ (unadjusted). (B) Accuracy as a function of population size for slow mixing, fast updates $v=0.1, \gamma=0.7$. Legend as (A). Main figure shows accuracy as a function of the rescaled leadership fraction $L N^{-\alpha}$ where $\alpha=-0.79$. Inset: Accuracy as a function of $L$ (unadjusted)

shown. If we rescale the leadership level these curves collapse and this is shown in the main figure; accuracy is therefore determined by the value of $L N^{-\alpha}$, where $\alpha$ is a fixed scaling exponent.

While data collapse is attained for both sets of parameter values, the scaling exponent required is different. To better visualize the nature of these exponents, we plot the leadership fraction that gives an accuracy level of 0.75 on log scale against population size in Fig. 3B. We observe a power law over close to four orders of magnitude. Further, we note the slope of these lines make a transition from $\sim-1$ to $\sim-0.5$ as the properties of the spatial simulation are modified.

While our parameter space is two-dimensional (the rate of opinion updates $\gamma$ and the mixing speed $v$ are varied), we find that both of these values combine to give a single quantity of interest; the number of novel observations made per opinion update (see Fig. 3A). If social mixing is very low, even if a large number of observations are made they will most likely be of a limited number of individuals, i.e. the same individual will be repeatedly sampled. However, if the update rate slows the independent sample number will increase, even for low mixing. We determine the average number of novel observations per opinion update by calculating the average frequency of changes in the identity of the nearest neighbor, i.e. the inverse of the time required for the nearest neighbor to be displaced sufficiently so that it is replaced by a third, closer, individual. We record this quantity for the parameter sets used and plot against the corresponding scaling exponent in Fig. 3D. A smooth monotonic transition is observed, from a linear scaling when there is one sample per update to an exponent of approximately $-1 / 2$ when there are multiple samples.

The numerical simulations show that the effect of space in our model is to restrict the average number of novel social interactions an individual has. This may be countered by reducing the rate of opinion updates. When these parameters combine so that the average number of distinct observations per update is close to one, accuracy is determined by the value of $L N$ (the absolute number of leaders). By reducing the rate of opinion switches or increasing the rate of mixing, the number of novel samples may be increased. As this number rises a transition occurs to a scaling regime where accuracy increases as the square root of $N$.

In order to analytically investigate the transition between scaling regimes we next formulate a mean-field version of the model that incorporates an explicit sample rate. 

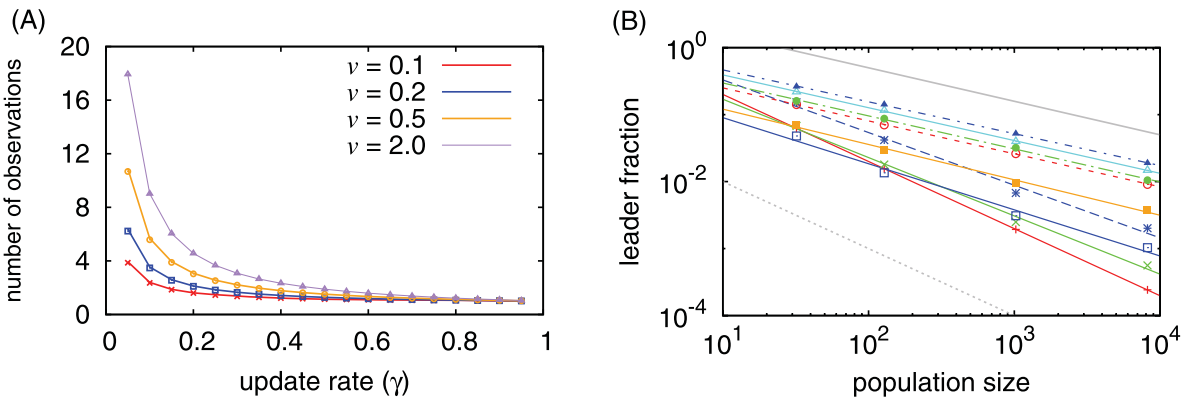

(C)

(D)
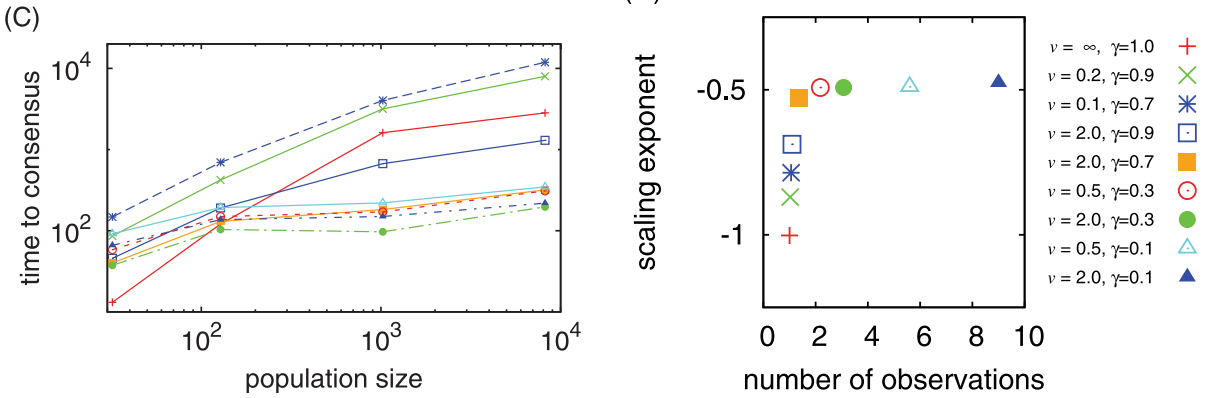

Fig. 3 Spatial simulations. (A) Average number of novel observations per opinion update as a function of mixing rate $(v)$ and individual update rate $(\gamma)$. To illustrate the invariance of this metric to group size, lines show values for $N=32$ while points show $N=8192$. (B) Scaling relations between accuracy and population size. Points show required informed individual fractions for an accuracy level of 0.75 for different population sizes and parameter values. $v=\infty$ is approximated by selecting a new nearest-neighbor at random every time step. Lines show a linear fit. Solid grey line and dashed grey line show slope of $f(N) \propto N^{-0.5}$ and $g(N) \propto N^{-1}$ respectively. (C) Average time to consensus for the accuracy rates shown in (B). Time is calculated as the number of time steps at which an opportunity for an update occurs and hence is invariant to mixing rate $(v)$ but not update probability $(\gamma)$. (D) Scaling exponents as a function of average number of novel observations per opinion update. Legend for $(\mathbf{B})$ and $(\mathbf{C})$ as in $(\mathbf{D})$

\section{A Well-Mixed Model with an Explicit Sample Count}

We reformulate our spatial model by removing the process of building up a set of observations over time. Instead an instantaneous sample of the opinion of $S$ individuals is taken. These sample individuals are chosen at random from the population and the size of the sample is explicitly set. Although we lose the local nature of the social interaction, the advantage of this approach is that the probability an individual transitions from one opinion to another can be calculated exactly. The opinion dynamics algorithm is then a reduced version of the full spatial simulations and is equivalent in this form to models of convention formation found in the economics literature [2].

Again, for clarity, we include here a summary of the algorithm used in simulations.

- At each timestep one individual is selected at random from the population.

- This individual will make a fixed number of observations $(S)$ of the population opinion, drawing at random with replacement.

- If within this sample more individuals are in the +1 state, the individual will switch to this state. 
(A)

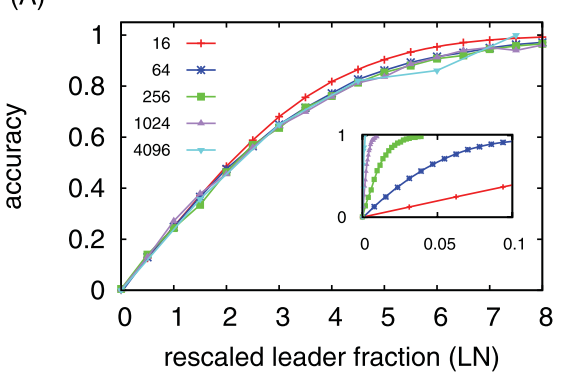

(C)

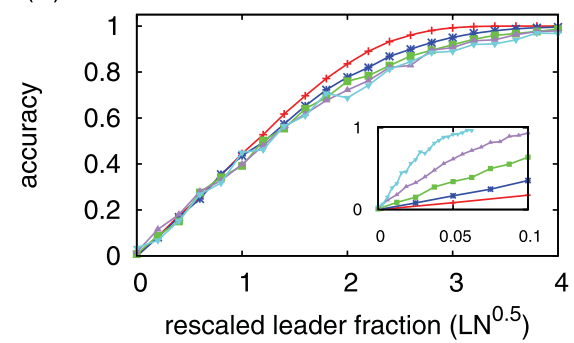

(B)

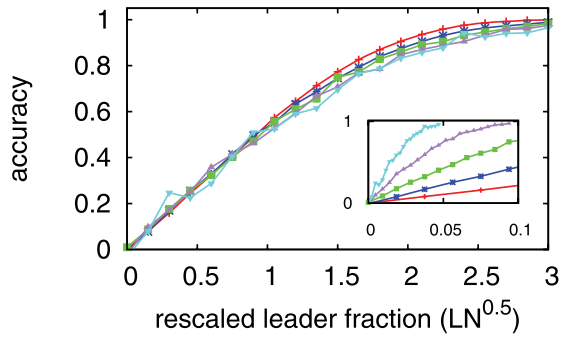

(D)

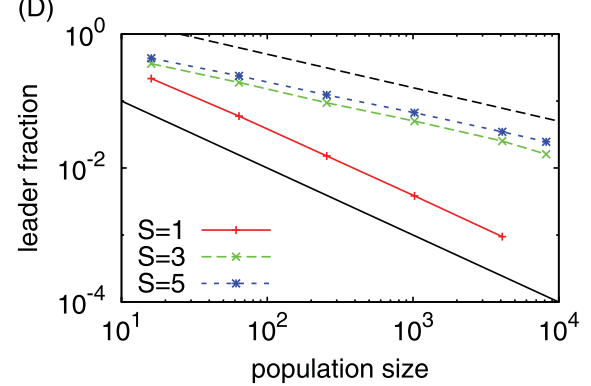

Fig. 4 Mean-field simulations. (A-C) Accuracy for mean-field simulations. Main figures show accuracy as a function of the rescaled informed individual fraction. Insets show unadjusted values. (A) Number of observations $(S)$ is $1,(\mathbf{B}) S=3,(\mathbf{C}) S=5$. Legend for (A-C) as A. (D) Leadership fraction required for accuracy of 0.75 . Lines show different scaling with various values of $S$

- However, if more individuals are in the -1 state, the individual will switch to this state with probability $(1-L)$. Otherwise it is considered an informed individual and will not switch to the incorrect state.

Simulations continue until all individuals share the same opinion and no further updates are possible. Numerical results from this model are shown in Fig. 4. As in the spatial simulations we observe a transition between scaling regimes as $S$ is varied. We next demonstrate this transition more formally.

\subsection{Mean-Field Analysis}

From Markov theory, an exact expression can be found for the probability of reaching the correct decision as a function of the transition rates, if the process is equated to a birth-death one step process. In this form the decision process takes place on a one-dimensional line where the current state of the system is defined as an integer that ranges from 0 to $N$ and represents the number of individuals with the correct opinion. The probability this one step process will ultimately reach the state $N$ (all correct) is [24]

$$
P(\text { correct decision })=\frac{1+\sum_{i=2}^{N / 2} \prod_{j=1}^{i-1} T(j / N)}{1+\sum_{i=2}^{N} \prod_{j=1}^{i-1} T(j / N)}
$$

where $T$ is the ratio of the probabilities of moving one step downwards (an individual switches from the preferred to non-preferred side) versus upwards (an opposite transition),

$$
T(x)=\frac{t^{-}(x)}{t^{+}(x)} .
$$


(See Appendix A for a derivation of Eq. (2) reproduced from [24].)

The functions $t^{ \pm}(x)$ are defined by the binomial probabilities of observing a majority for a given side after making $S$ samples of the population, given the overall fraction of individuals in the preferred state is $j / N$ :

$$
\begin{gathered}
t^{-}\left(\frac{j}{N}\right)=(1-L)\left(\frac{j}{N}\right)\left(1-\sum_{k=0.5(S+1)}^{S}\left(\begin{array}{l}
S \\
k
\end{array}\right)\left(\frac{j}{N}\right)^{k}\left(1-\frac{j}{N}\right)^{S-k}\right) \\
t^{+}\left(\frac{j}{N}\right)=\left(1-\frac{j}{N}\right)\left(\sum_{k=0.5(S+1)}^{S}\left(\begin{array}{l}
S \\
k
\end{array}\right)\left(\frac{j}{N}\right)^{k}\left(1-\frac{j}{N}\right)^{S-k}\right)
\end{gathered}
$$

To avoid an equal number of observations of each opinion we assume that $S$ is always odd; we lose no generality by making this assumption since an even sample size of $S$ is equivalent to a sample size of $S-1$, if an appropriate unbiased tie-break rule is introduced.

In the well-mixed limit Eq. (2) is exact, however its discrete nature means it is largely resistant to manipulation. To counter this, we introduce a continuous approximation to this equation as follows.

For the product term of Eq. (2), we take logarithms, then employ the mid-point method to substitute the summation for a definite integral,

$$
\begin{aligned}
\prod_{j=1}^{i-1} T(j / N) & =\exp \sum_{j=1}^{i-1} \ln T(j / N) \\
& \simeq \exp \left[N \int_{0.5 / N}^{(i-0.5) / N} \ln T(x) d x\right]
\end{aligned}
$$

where we have introduced the continuous variable $x=j / N$.

In a similar fashion, the denominator of Eq. (2), may be written as,

$$
\begin{aligned}
1 & +\sum_{i=2}^{N} \exp \left[N \int_{0.5 / N}^{(i-0.5) / N} \ln T(x) d x\right] \\
& =\sum_{i=1}^{N} \exp \left[N \int_{0.5 / N}^{(i-0.5) / N} \ln T(x) d x\right] \\
& \simeq N \int_{0.5 / N}^{(N+0.5) / N} \exp \left[N \int_{0.5 / N}^{z-(0.5 / N)} \ln T(x) d x\right] d z
\end{aligned}
$$

Neglecting the $\mathcal{O}(1 / N)$ terms in the limits of this equation, and applying the same procedure to the numerator of Eq. (2) leaves,

$$
P(\text { correct decision })=\frac{\int_{0}^{0.5} \exp [N \phi(x)] d x}{\int_{0}^{1} \exp [N \phi(x)] d x}
$$

where, following [25], we define the effective potential for the system as

$$
\phi(x)=\int_{0}^{x} \ln T(z) d z .
$$

We next attain an approximation for the potential $\phi(x)$. To do this we perform a Taylor expansion around $x=0.5$, under the assumption that the dynamics are largely determined by the properties of the central region,

$$
\phi(x) \simeq \phi(0.5)+(x-0.5) \ln T(0.5)+\left.\frac{1}{2}(x-0.5)^{2} \frac{d \ln T(x)}{d x}\right|_{x=0.5}
$$


The first term is simply a constant that will later cancel. The second term is determined by the sampling and switching probabilities defined by Eqs. (3), (4), and (5), which, if we consider $S$ as a variable, may be expressed as a function of $x$ and $S$ as,

$$
T(x, S)=\frac{x(1-B(x, S))(1-L)}{(1-x) B(x, S)}
$$

where

$$
B(x, S)=\sum_{k=0.5(S+1)}^{S}\left(\begin{array}{l}
S \\
k
\end{array}\right)(x)^{k}(1-x)^{S-k} .
$$

Since $B(0.5, S)=0.5$,

$$
\ln T(0.5, S)=\ln (1-L)
$$

For the third term of Eq. (10),

$$
\begin{aligned}
\left.\frac{\partial \ln T(x, S)}{\partial x}\right|_{x=0.5} & =\left.\frac{1}{T(0.5, S)} \frac{\partial T}{\partial x}\right|_{x=0.5} \\
& =\left.\frac{\partial}{\partial x}\left(\frac{x(1-B(x, S))}{(1-x) B(x, S)}\right)\right|_{x=0.5} .
\end{aligned}
$$

We employ product and quotient rules to find,

$$
\left.\frac{\partial \ln T(x, S)}{\partial x}\right|_{x=0.5}=4-\left.4 \frac{\partial B}{\partial x}\right|_{x=0.5}
$$

An exact expression for the derivative in this equation is

$$
\left.\frac{\partial B}{\partial x}\right|_{x=0.5}=\frac{1}{2}^{S-1}\left(\begin{array}{c}
S-1 \\
(S-1) / 2
\end{array}\right) S
$$

where the term in parentheses is a binomial coefficient. Further, for large $S$ a simpler expression may be found by applying Stirling's approximation to the factorial terms,

$$
\left.\frac{\partial B}{\partial x}\right|_{x=0.5} \simeq \sqrt{\frac{2 S}{\pi}}
$$

(See Appendix B for the derivation of Eqs. (16) and (17).)

Combining these results into Eq. (10) gives

$$
\phi(x) \simeq \begin{cases}C+\ln (1-L) x+\left(2-\frac{1}{2}^{S-2}\left(\begin{array}{c}
S-1 \\
(S-1) / 2
\end{array}\right) S\right)(x-0.5)^{2} & \text { for all } S \\
C+\ln (1-L) x+\left(2-\sqrt{\frac{8 S}{\pi}}\right)(x-0.5)^{2} & \text { for } S \gg 1\end{cases}
$$

where $C$ is a constant. A comparison of this result with computationally calculated values of the true potential are shown in Fig. 5A.

Substituting this result into the integral of Eq. (8) gives,

$$
\int \exp \left(C+\ln (1-L) N x+\left(2-\frac{1}{2}^{S-2}\left(\begin{array}{c}
S-1 \\
(S-1) / 2
\end{array}\right) S\right) N(x-0.5)^{2}\right) d x .
$$

While this integral may be solved, it is worth noting at this point that when one observation is made per opinion update, i.e. the potential is linear and there is no positive feedback in the decision process, accuracy is determined solely by the value of $L N$ (or $\ln (1-L) N$ for large $L$ ). Thus, when $S=1$ and the leadership fraction is small, it is the absolute number 

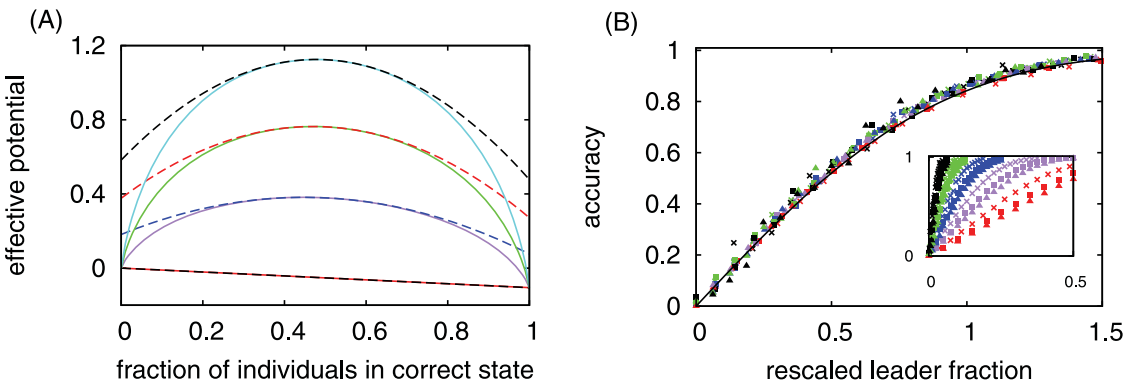

Fig. 5 (A) Approximation to the effective potential. Solid lines show the effective potential defined by Eq. (9), calculated using Mathematica. Dashed lines show Eq. (18). Number of samples shown are (from top) $S=7, S=5, S=3, S=1$. The constant $C$ has been found numerically for illustration. (B) Analytical success rate for $S>1$. The solid line shows Eq. (23). Points are numerical results plotted as a function of the rescaled leadership fraction suggested by Eq. (23). Values shown are for $S=7$ (triangles), $S=5$ (squares), $S=3$ (crosses), and for group sizes 4096 (black), 1024 (green), 256 (blue), 64 (purple), 16 (red). Inset shows unadjusted results

of informed individuals that is the parameter of interest. Further, the collective decision in this regime becomes equivalent to the well-known Gambler's ruin problem, which may be solved exactly, as described in [26].

When $S>1$, as the integral has a closed form solution, we may now write the probability of reaching the correct decision as

$$
\frac{\operatorname{Erf}\left(\sqrt{\frac{N}{8(\sqrt{2 S / \pi}-1)}} L\right)-\operatorname{Erf}\left(\sqrt{\frac{N}{8(\sqrt{2 S / \pi}-1)}} L\left(1-\frac{2(\sqrt{2 S / \pi}-1)}{L}\right)\right)}{\operatorname{Erf}\left(\sqrt{\frac{N}{8(\sqrt{2 S / \pi}-1)}} L\left(1+\frac{2(\sqrt{2 S / \pi}-1)}{L}\right)\right)-\operatorname{Erf}\left(\sqrt{\frac{N}{8(\sqrt{2 S / \pi}-1)}} L\left(1-\frac{2(\sqrt{2 S / \pi}-1)}{L}\right)\right)}
$$

Since $S>1$ we have used Stirling's approximation to simplify the factorial terms, and further assumed $L \ll 1$ so that $\ln (1-L) \simeq-L$. (For $L \gg 0$ the leadership fraction in the equation above must be replaced with $-\ln (1-L)$.)

When $S>1$ and $L$ is small, i.e.

$$
\frac{(\sqrt{2 S / \pi}-1)}{L}>1
$$

sociality will dominate and three of the four error functions contained in Eq. (20) tend to unity, leaving

$$
\frac{\operatorname{Erf}\left(\sqrt{\frac{N}{8(\sqrt{2 S / \pi}-1)}} L\right)+1}{2} .
$$

Accuracy as defined previously on a scale from zero to one is therefore

$$
\operatorname{Erf}\left(\sqrt{\frac{N}{8(\sqrt{2 S / \pi}-1)}} L\right)
$$

This value is plotted in Fig. 5B for comparison of the numerical simulations to the analytical result. Hence, we see in this regime accuracy scales as $\sqrt{N}$.

This result holds even when the initial condition is not an evenly balanced opinion. If a given side begins in the majority then Eq. (23) becomes

$$
\operatorname{Erf}\left(\sqrt{N}\left(\frac{L}{\sqrt{8(\sqrt{2 S / \pi}-1)}}+\sqrt{(2 \sqrt{2 S / \pi}-1)}\left(x_{I C}-0.5\right)\right)\right)
$$


where $x_{I C}$ is the initial fraction of the population adopting the correct opinion. Thus the scaling relation remains, however any asymmetry in the initial conditions affects the overall level of accuracy and this effect is stronger when sociality is high.

Finally, we note Eq. (21) provides valuable insight into the nature of the transition between scaling regimes when the expected number of observations between opinion changes varies continuously. If we consider the social feedback effect as a continuous variable, $\epsilon$, which is 0 when $S=1$ and increases monotonically with $S$, then we may write the condition for $\sqrt{N}$ scaling as $\epsilon / L>1$. In the limit of $\epsilon / L \rightarrow 0$ the Gambler's ruin process is recovered from Eq. (20) and accuracy is determined by $L N$. Hence for any non-zero value of $\epsilon$, accuracy will scale with $L N$ until the required leadership fraction is of $\mathcal{O}(\epsilon)$. At this point the degree of curvature in the potential becomes significant and a transition occurs to the $\sqrt{N}$ scaling regime.

\section{Discussion}

Through our binary choice model we have demonstrated how spatial mixing, memory and sociality influence the dynamics of collective decisions. We have shown that if there exists an imposed bias in the decision-making process, meaning one option is the correct choice, then the likelihood of selecting this option increases with the size of the group. This effect is in agreement with previous individual-based simulations of leadership [16], and theoretical models that assume centralized counting of votes or do not explicitly provide an aggregation mechanism [11, 14].

By recovering these results in our model we show this to be a general property and we are further able to reconcile the disparity between the individual-based spatial model, where accuracy appears to depend on the absolute number of leaders $(L N)$, and the $\sqrt{N}$ scaling that underpins both Condorcet's jury theorem [11] and the 'many wrongs' hypothesis [14]. We observe that the absolute number of informed individuals is the variable of interest when there is approximately one novel observation per opinion change. The decision process in this scenario is a neutral (or diffusive) process since there is a balance between the probability of selecting an individual of a particular type to switch and of selecting an opposing type to observe. This results in constant transition probabilities toward or away from consensus regardless of the numbers holding each opinion. The information content therefore has a consistent effect and the result is equivalent to a biased random walk with absorbing boundaries. Absorption probabilities in this case can be found exactly and it is easy to show that accuracy scales with the absolute number of leaders. In this situation, the role of space is to increase the time it takes to reach consensus and enable greater accuracy as the group size increases.

Increasing the effect of sociality by increasing the number of observations introduces a positive feedback, such that when one opinion begins to dominate it becomes increasingly likely that it will be the eventual consensus viewpoint. In this regime, the decisions become faster and less accurate, and the $\sqrt{N}$ scaling associated with the central limit theorem is recovered, suggesting no explicit aggregation procedure is necessary to observe this property. It is worth noting that slowing the decisions of individuals has an opposing effect at the collective level. When an individual makes a decision quickly, i.e. after only a small number of observations, the process is more random and the collective decision becomes slower. Conversely, if individual updates are less frequent a more accurate assessment of the current consensus is attained, therefore a much faster collective decision is reached, since when a given side becomes the majority this is more readily observed and the positive feedback toward that side is stronger. 
Investigating how these properties of social interaction affect opinion dynamics is important for both human and animal social groups. For social animals, studying decision-making will enhance our understanding of the collective processes that are vital for their survival. Much focus in recent times has been on the performance improvements seen as group sizes increase, but the negative consequence of these phenomena are compounding Allee effects [27] that lead to faster rates of decline as numbers reduce. The scaling relations observed here are not only mathematical quantities but, in populations where different individuals have access to environmental information, they suggest how the relative weightings of leaders and followers need to shift in order to maintain function as group sizes reduce.

Although the spatially explicit model represents collective decision-making by individuals moving within a two-dimensional space, the mean-field model demonstrates that our results are potentially more general. While the nature of the localized interaction in our model discounts formal voting processes, such as in a democratic election, our results may be applicable to decentralized socially-mediated decisions in human groups. For example, there may be direct analogies with binary choices that diffuse through dynamic social networks, such as adopting novel technologies, political or social unrest, stock market valuations, or rejecting commonly-held but erroneous beliefs [27-32]. In many of these scenarios there is a direct cost associated with going against the majority viewpoint [33], hence imitation is a rational behavior and can be expected to be widespread.

For both human and non-human animals research has consistently demonstrated the degree to which social factors influence choices and actions [34-36]. It is therefore clear that collective decisions are not only determined by the direct response of individuals to the information they receive, but are also dramatically influenced by local interactions, the nature of which may entirely determine the accuracy of the decision-making process.

Acknowledgements This research was supported by Army Research Office Grant No. W911NG-11-10385, Office of Naval Research Grant No. N00014-09-1-1074, and National Science Foundation Award PHY0848755. The authors wish to thank Andrew Hartnett and Albert Kao for comments on the manuscript, and Peter R. Kramer of the Rensselaer Polytechnic Institute, New York, for directing us toward the derivation outlined in Appendix A.

\section{Appendix A: Derivation of Absorption Probability}

Absorption probabilities for the Markov process may be calculated exactly as shown in [24]. This derivation proceeds as follows. We firstly define the number of individuals in the correct state as a one step Markov process. Each state is therefore an integer value ranging from 0 (all individuals are incorrect) to $N$ (all individuals are correct). As each boundary is an absorbing state, the probability of absorption $U_{i}$ in state $N$ (all correct) from state $i$ satisfies the equation

$$
U_{i}= \begin{cases}0 & \text { for } i=0 \\ t_{i}^{-} U_{i-1}+\left(1-t_{i}^{-}-t_{i}^{+}\right) U_{i}+t_{i}^{+} U_{i+1} & \text { for } 1 \leq i \leq N-1 \\ 1 & \text { for } i=N\end{cases}
$$

where $t^{+}$and $t^{-}$are the probabilities of transitioning to the state one step above or below respectively. Rewriting the expression for the interior states gives,

$$
t_{i}^{-}\left(U_{i-1}-U_{i}\right)+t_{i}^{+}\left(U_{i+1}-U_{i}\right)=0
$$

We now define

$$
V_{i}=U_{i}-U_{i-1}
$$


so that

$$
\begin{gathered}
-t_{i}^{-} V_{i}+t_{i}^{+} V_{i+1}=0 \\
V_{i+1}=\frac{t_{i}^{-}}{t_{i}^{+}} V_{i} .
\end{gathered}
$$

All values of $V$ can therefore be calculated from $V_{1}$,

$$
V_{i}=\left(\prod_{k=1}^{i-1} \frac{t_{k}^{-}}{t_{k}^{+}}\right) V_{1}
$$

To solve for $V_{1}$ we use the relation

$$
\sum_{i=1}^{N} V_{i}=U_{N}-U_{0}
$$

that arises from the definition of $V$ and the cancelling of interior terms of the series. From Eqs. (29) and (30),

$$
V_{1}+\sum_{i=2}^{N}\left(\prod_{k=1}^{i-1} \frac{t_{k}^{-}}{t_{k}^{+}}\right) V_{1}=1
$$

therefore,

$$
V_{1}=\left(1+\sum_{j=2}^{N}\left(\prod_{k=1}^{j-1} \frac{t_{k}^{-}}{t_{k}^{+}}\right)\right)^{-1}
$$

Again by definition,

$$
U_{i}=\sum_{j=1}^{i} V_{j}+U_{0}
$$

which, in combination with the expression for $V_{1}$ and $V_{i}$, and the value of $U_{0}=0$, leads to

$$
U_{i}=\frac{1+\sum_{j=2}^{i}\left(\prod_{k=1}^{j-1} \frac{t_{k}^{-}}{t_{k}^{+}}\right)}{1+\sum_{j=2}^{N}\left(\prod_{k=1}^{j-1} \frac{t_{k}^{-}}{t_{k}^{+}}\right)} .
$$

The quantity of interest is the absorption probability of the correct state when initially the system begins with equal numbers of individuals with each opinion, i.e. $U_{0.5 N}$. This value is

$$
\frac{1+\sum_{j=2}^{0.5 N}\left(\prod_{k=1}^{j-1} \frac{t_{k}^{-}}{t_{k}^{+}}\right)}{1+\sum_{j=2}^{N}\left(\prod_{k=1}^{j-1} \frac{t_{k}^{-}}{t_{k}^{+}}\right)} .
$$

Appendix B: Derivation of $\frac{\partial B}{\partial x}$

Given the binomial probability

$$
B(x, S)=\sum_{k=0.5(S+1)}^{S}\left(\begin{array}{l}
S \\
k
\end{array}\right)(x)^{k}(1-x)^{S-k}
$$


we wish to find

$$
\left.\frac{\partial B}{\partial x}\right|_{x=0.5}
$$

We take derivatives, then rearrange to give

$$
\begin{aligned}
\left.\frac{\partial B}{\partial x}\right|_{x=0.5} & =\sum_{k=0.5(S+1)}^{S} \frac{1}{2}^{S-1}\left(\begin{array}{l}
S \\
k
\end{array}\right)(2 k-S) \\
& =\frac{1}{2}{ }^{S-1}\left(2 \sum_{k=0.5(S+1)}^{S} k\left(\begin{array}{l}
S \\
k
\end{array}\right)-S \sum_{k=0.5(S+1)}^{S}\left(\begin{array}{l}
S \\
k
\end{array}\right)\right)
\end{aligned}
$$

Since

$$
\sum_{k=0}^{n}\left(\begin{array}{l}
n \\
k
\end{array}\right)=2^{n}
$$

and the binomial coefficients are symmetric, this reduces to

$$
\begin{aligned}
\left.\frac{\partial B}{\partial x}\right|_{x=0.5} & =\frac{1}{2}^{S-1}\left[2 S\left(2^{S-2}+\frac{1}{2}\left(\begin{array}{c}
S-1 \\
(S-1) / 2
\end{array}\right)\right)-S 2^{S-1}\right] \\
& =\frac{1}{2} S\left(\begin{array}{c}
S-1 \\
(S-1) / 2
\end{array}\right) .
\end{aligned}
$$

This is an exact result, however a simpler expression for large $S$ may be obtained by introducing Stirling's approximation for the binomial coefficients.

Define $T=(S-1) / 2$, then

$$
\begin{aligned}
\left(\begin{array}{c}
S-1 \\
(S-1) / 2
\end{array}\right) & =\frac{(2 T) !}{(T !)(T !)} \\
& \simeq \frac{\sqrt{4 \pi T}(2 T / e)^{2 T}}{2 \pi T(T / e)^{2 T}} \\
& \simeq 2^{2 T} \sqrt{\frac{1}{\pi T}} \\
& \simeq 2^{S-1} \sqrt{\frac{2}{\pi(S-1)}}
\end{aligned}
$$

Therefore,

$$
\begin{aligned}
\left.\frac{\partial B}{\partial x}\right|_{x=0.5} & \simeq \frac{1}{2} S \sqrt{\frac{S-1}{\pi(S-1)}} 2^{S-1} \\
& \simeq \sqrt{\frac{2 S^{2}}{\pi(S-1)}} \\
& \simeq \sqrt{\frac{2 S}{\pi}}
\end{aligned}
$$




\section{References}

1. Torney, C.J., Levin, S.A., Couzin, I.D.: Proc. Natl. Acad. Sci. 107, 20394 (2010)

2. Young, H.P.: Econometrica 61(1), 57-84 (1993)

3. Liggett, T.M.: Stochastic Interacting Systems: Contact, Voter, and Exclusion Processes vol. 324. Springer, Berlin (1999)

4. Castellano, C., Fortunato, S., Loreto, V.: Rev. Mod. Phys. 81, 591 (2009)

5. Vicsek, T., Czirók, A., Ben-Jacob, E., Cohen, I., Shochet, O.: Phys. Rev. Lett. 75, 1226 (1995)

6. Buhl, J., et al.: Science 312, 1402 (2006)

7. Galam, S.: Sociophysics: A Physicist's Modeling of Psycho-Political Phenomena. Springer, Berlin (2012)

8. Anderson, P.W.: Science 177, 393 (1972)

9. Mobilia, M.: Phys. Rev. Lett. 91, 028701 (2003)

10. Sood, V., Redner, S.: Phys. Rev. Lett. 94, 178701 (2005)

11. Condorcet, M.: In: Baker, K.M. (ed.) Reprinted in Condorcet: Selected Writings, vol. 33 (1976)

12. Galton, F.: Nature 75, 450 (1907)

13. Grünbaum, D.: Evol. Ecol. 12, 503 (1998)

14. Simons, A.: Trends Ecol. Evol. 19, 453 (2004)

15. Surowiecki, J.: The Wisdom of Crowds. Anchor, New York (2005)

16. Couzin, I.D., Krause, J., Franks, N.R., Levin, S.A.: Nature 433, 513 (2005)

17. Torney, C.J., Neufeld, Z., Couzin, I.D.: Proc. Natl. Acad. Sci. 106, 22055 (2009)

18. Berdahl, A., Torney, C.J., Ioannou, C.C., Faria, J., Couzin, I.D.: Science 339(6119), 574-576 (2013)

19. Couzin, I.D., et al.: Science 334, 1578 (2011)

20. Xie, J., et al.: Phys. Rev. E 84, 011130 (2011)

21. Huse, G., Railsback, S., Ferono, A.: J. Fish Biol. 60(3), 571-582 (2002)

22. Cavagna, A., Queiros, S., Giardina, I., Stefanini, F., Viale, M.: arXiv:1206.4434 (2012)

23. Neufeld, Z., López, C., Haynes, P.H.: Phys. Rev. Lett. 82, 2606 (1999)

24. Hoel, P., Port, S., Stone, C.: Introduction to Stochastic Processes. Houghton Mifflin, Boston (1972)

25. Doering, C.R., Sargsyan, K.V., Sander, L.M.: Multiscale Model. Simul. 3, 283-299 (2006)

26. Feller, W.: An Introduction to Probability Theory and Its Applications vol. 2. Wiley, New York (2008)

27. Saavedra, S., Hagerty, K., Uzzi, B.: Proc. Natl. Acad. Sci. 108, 5296 (2011)

28. Banerjee, A.V.: Q. J. Econ. 107, 797 (1992)

29. Arthur, W.B.: Econ. J. 99, 116 (1989)

30. Bikhchandani, S., Hirshleifer, D., Welch, I.: J. Polit. Econ. 100, 992-1026 (1992). http://www.jstor.org/ stable/2138632

31. Lohmann, S.: World Polit. 47, 42 (1994)

32. Granovetter, M.: Am. J. Sociol. 83(6), 1420-1443 (1978)

33. Young, H.: Individual Strategy and Social Structure: An Evolutionary Theory of Institutions. Princeton University Press, Princeton (2001)

34. Dall, S., Giraldeau, L., Olsson, O., McNamara, J., Stephens, D.: Trends Ecol. Evol. 20,187 (2005)

35. Salganik, M., Dodds, P., Watts, D.: Science 311, 854 (2006)

36. Faria, J.J., Krause, S., Krause, J.: Behav. Ecol. 21, 1236 (2010) 